\title{
Comparative analysis of steady state heat transfer in a TBC and functionally graded air cooled gas turbine blade
}

\author{
NILANJAN COOMAR and RAVIKIRAN KADOLI* \\ Mechanical Engineering Department, National Institute of Technology \\ Karnataka, Surathkal, Srinivasnagar 575025 \\ e-mail: rkkadoli@rediffmail.com; coomar160@yahoo.co.in
}

MS received 4 December 2007; revised 23 December 2009; accepted 7 January 2010

\begin{abstract}
Internal cooling passages and thermal barrier coatings (TBCs) are presently used to control metal temperatures in gas turbine blades. Functionally graded materials (FGMs), which are typically mixtures of ceramic and metal, have been proposed for use in turbine blades because they possess smooth property gradients thereby rendering them more durable under thermal loads. In the present work, a functionally graded model of an air-cooled turbine blade with airfoil geometry conforming to the NACA0012 is developed which is then used in a finite element algorithm to obtain a non-linear steady state solution to the heat equation for the blade under convection and radiation boundary conditions. The effects of external gas temperature, coolant temperature, surface emissivity changes and different average ceramic/metal content of the blade on the temperature distributions are examined. Simulations are also carried out to compare cooling effectiveness of functionally graded blades with that of blades having TBC. The results highlight the effect of including radiation in the simulation and also indicate that external gas temperature influences the blade heat transfer more strongly. It is also seen that graded blades with about $70 \%$ ceramic content can deliver better cooling effectiveness than conventional blades with TBC.
\end{abstract}

Keywords. Turbine blade cooling; finite element method (FEM); functionally graded materials (FGM); thermal barrier coating (TBC).

\section{Introduction}

Increase in the turbine entry temperature (TET) is always a design goal for gas turbines because increases in TET result in an increase in output and improvement in efficiency. However, temperatures above $900^{\circ} \mathrm{C}$, corrosion problems assume significance and protective coatings have to be used. Ceramic-based thermal barrier coatings (TBC) are employed to provide protection to the blade metal in this extreme high-temperature environment. It is well-known

*For correspondence 
that use of TBC coatings can be plagued by durability issues due to phase instability, thermal instability and adherence problems at elevated temperatures and in corrosive environments. TBCs also tend to spall on thermal cycling because of thermal expansion mismatch with the superalloy substrate or because of erosion/impact caused by particles in the high-velocity combustion gases (Boyce 2002). While blades with TBCs are the norm today, functionally graded materials (FGMs) are a relatively new class of materials that can operate without the above problems in high-temperature environments and can be considered as potential material for making gas turbine blades. Typically, FGMs are composite materials made of a mixture of ceramic and metal. In FGMs the volume fraction of the metal and ceramic are gradually varied so that mechanical properties of the body change smoothly and continuously through its volume, unlike the case for a TBC coated metal part. FGM compositions can be readily tailored to gain selective advantages from the properties of the individual components. The ceramic provides the high temperature resistance, and its coefficient of thermal expansion is also small so that for a given heat flux it experiences less thermal strains and stresses. At the same time, the ductile metal constituent prevents fracture due to stresses caused by sudden thermal shocks. The smoothly varying material property gradients eliminate interface mismatch problems (Lee et al 1996). For these reasons FGMs are better placed than TBCs to handle concerns regarding durability.

Heat transfer in combustor linings, nozzles, buckets and blade discs in gas turbines have received much attention (Garg 2002). Lakshminarayana (1995) has examined the physics of such heat transfer mechanisms in detail. Computational fluid dynamics (CFD) tools have been typically applied for predicting flow and heat transfer in cooling passages of turbine blades (Iacovides \& Launder 1995). Various experimental, theoretical and numerical approaches have been implemented for the study of blade temperatures and heat transfer (Bunker et al 2000; Aydin et al 2002; Carcasi \& Facchini 1996). Asok Kumar \& Kale (2002) simulated the steady state heat transfer using the finite element method (FEM) in a metal turbine blade coated with $\mathrm{TBC}$, incorporating both convection and radiation heat transfer. They studied the effects on the overall heat transfer of applying partial TBC coatings in critical areas and of uncertainties in surface emissivities and external heat transfer coefficients. They concluded that selective TBC coating application can be effective and that uncertainty of convection heat transfer coefficient has negligible effect on the heat transfer in the presence of radiation and/or TBC. For FGMs aspects of heat transfer, thermomechanical response and crack formation and growth have been analysed and reported. These heat transfer studies for FGMs have been conducted by various researchers but these have been mostly carried out for regular geometries like plates, beams, cylinders and shells (Berger et al 2005; Reddy \& Chin 1998; Praveen \& Reddy 1999; Oh et al 2003; Wang et al 2004; Fujimoto \& Noda 2000). The problem of obtaining the temperature distribution within the body of a turbine blade assuming it is made of FGM and having temperature-dependent material properties and radiation heat transfer has not been considered before. While heat transfer studies exist for air-cooled turbine blades with TBC coating (Asok Kumar \& Kale 2002), hardly any studies have focused on making a comparison between the heat transfer in an air-cooled metal blade with just an outer layer of TBC and an air-cooled blade made of FGM.

In the present work, steady state heat transfer in an air-cooled functionally graded turbine blade is studied using a finite element code. For modelling the blade with FGM, a procedure based on analytical geometry is used to vary the volume fraction of the metal and ceramic as per a power law distribution over the blade domain. Temperature distribution of the same blade without FGM but having a layer of TBC is also computed and the two temperature distributions 
are compared. The effect of the main variables that influence the heat transfer problem, namely, TET, coolant temperature and surface emissivity are also studied and analysed.

\section{Formulation and computational background}

A FEM-based numerical scheme was developed for a two-dimensional turbine blade, which is taken to be the symmetric NACA0012 airfoil. The salient features of the FEM formulation are as follows.

\subsection{Heat transfer model for FGM turbine blade}

The governing equation for two-dimensional steady state heat transfer is given by:

$$
\frac{\partial}{\partial x}\left(k \frac{\partial T}{\partial x}\right)+\frac{\partial}{\partial y}\left(k \frac{\partial T}{\partial y}\right)=0 .
$$

In the FEM formulation, the material properties are assumed to be isotropic within each element but since, for the FGM blades, the volume fraction of ceramic and metal varies from one element to the next, there will be a spatial variation of material properties over the blade as a whole. Thermal conductivities are taken to be dependent on temperature. Heat transfer to the blade occurs, firstly, due to convection from the free stream hot gases. This has been modelled by assuming a constant free stream temperature, same as TET, and a local heat transfer coefficient which varies along both the pressure and the suction surfaces in the same manner. Fluid viscosity sets up a velocity gradient and brings the fluid to rest near the surface of the airfoil, impeding the heat transfer. This effect is exacerbated as the decelerated boundary layer region grows thicker further downstream of the airfoil surface due to the retarding action of the viscous fluid near the wall extending further into the main fluid domain. Thus, the maximum value of the external heat transfer coefficient $\left(h_{\text {ext }}\right)$ will occur near the leading edge where the boundary layer is thinnest. This kind of spatial variation of $h_{\text {ext }}$ is typically seen in experimental studies (Wang et al 2007). However, in the absence of actual experimental data for the spatial variation of $h_{\text {ext }}$ for a NACA0012 airfoil immersed in the hot gases in a typical gas turbine flow path, we propose to model this expected spatial variation of the $h_{\text {ext }}$ through a simplified relationship of the form of:

$$
h_{\mathrm{ext}}=h_{\mathrm{te}}+\left(h_{\mathrm{le}}-h_{\mathrm{te}}\right) e^{-4(x l / c)^{3}} .
$$

Here $h_{\text {te }}$ taken as $1000 \mathrm{~W} / \mathrm{m}^{2} \mathrm{~K}$ and $h_{\text {le }}$ taken as $4000 \mathrm{~W} / \mathrm{m}^{2} \mathrm{~K}$. The spatial variation of $h_{\text {ext }}$ is plotted in figure 1 . The second heat transfer mechanism on both the external pressure and suction airfoil surfaces that must be taken into account is the radiation from the hot gases and the other surfaces. The radiation environment is assumed to be an all-enclosing large surface whose temperature is the same as the TET. Further, the blade is assumed to not seen itself and the radiation is considered diffuse.

In this convection heat transfer problem there are two surfaces, where two Neumann boundary conditions are needed. The boundary condition at the internal surfaces (Surface $S_{2}$, figure 2), involving only convection is:

$$
k\left(\frac{\partial T}{\partial x}\right) l_{x}+k\left(\frac{\partial T}{\partial y}\right) l_{y}+h_{\mathrm{int}}\left(T_{s}-T_{c}\right)=0 .
$$




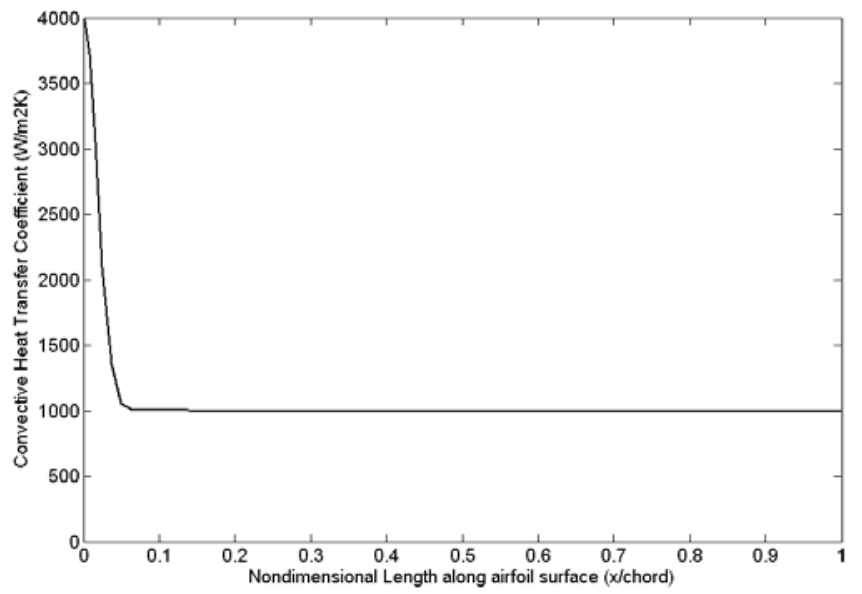

Figure 1. Typical surface variation of heat transfer coefficient for the airfoil representing the gas turbine blade (both pressure and suction surfaces).

The boundary condition at the external pressure and suction surfaces (Surface $S_{1}$, figure 2), including convection and radiation, is:

$$
k\left(\frac{\partial T}{\partial x}\right) l_{x}+k\left(\frac{\partial T}{\partial y}\right) l_{y}+h_{\mathrm{ext}}\left(T_{\infty}-T\right)_{s}+h_{r}\left(T_{\infty}-T_{s}\right)=0 .
$$

Note that in (4), the radiation heat transfer at the external surface has been reduced to just an equivalent convection boundary condition. The nonlinearity is incorporated through an additional temperature dependent radiation coefficient, $h_{r}$. This nonlinear radiation heat transfer coefficient can be easily seen to be (Huebner et al 1995):

$$
h_{r}=\varepsilon \sigma\left(T_{\infty}^{2}+T_{s}^{2}\right)\left(T_{\infty}+T_{s}\right) .
$$

Gas turbine blade alloy material conductivities are usually low, so conduction along the blade can be neglected. However, the cooling air temperature increases as it flows through the blade and picks up heat. These three-dimensional complexities have been ignored and the coolant is assumed to be at the same temperature in all the passages along with a uniform heat transfer coefficient in each passage which implies that $h_{\text {int }}$ used in (3) is constant and the same for all the internal surfaces.

\subsection{Finite element formulation}

The finite element formulation was developed using the Galerkin weighted residual method (Lewis et al 2004). Thus, choosing the weighting functions to be the same as the shape functions, we require that

$$
\int_{v} N_{i}\left[\frac{\partial}{\partial x}\left(k \frac{\partial T^{e}}{\partial x}\right)+\frac{\partial}{\partial y}\left(k \frac{\partial T^{e}}{\partial y}\right)\right] d v=0,
$$

$i=1,2,3, \ldots, n$. By using Green's theorem for expanding the terms in the above integral, the element equations are obtained in the matrix form

$$
\mathbf{K T}=\mathbf{F} .
$$




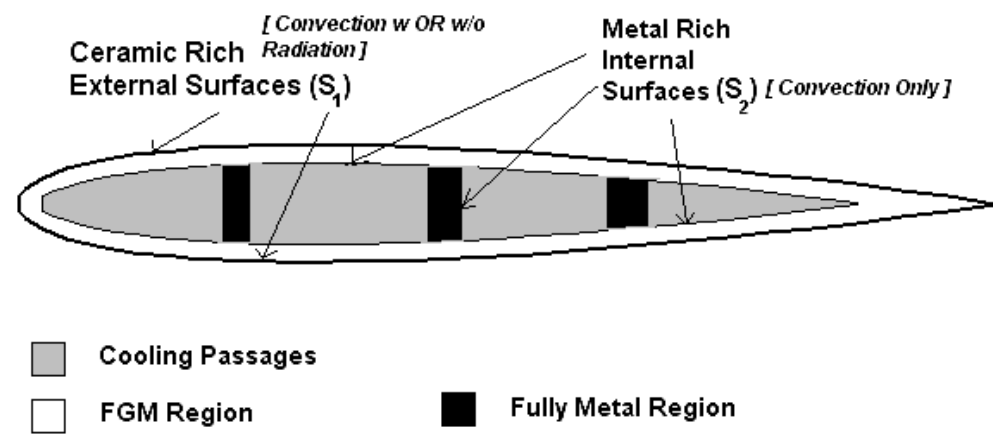

Figure 2. FGM model of turbine blade with profile of NACA0012 airfoil.

Both the stiffness matrix $\mathbf{K}$ and the load vector $\mathbf{F}$ incorporate contributions from the radiation and convection boundary conditions. Linear triangular elements are used in the FEM discretisation. The resultant global system of equations is a set of nonlinear algebraic equations. The nonlinearity arises due to the radiation term and also due to the temperature-dependent material thermal conductivities. This nonlinear system is solved iteratively to a desired degree of convergence using the Picard scheme, where both the stiffness matrix and the load vector are assumed to be known from the previous iteration (Reddy 1993).

\subsection{Mesh generation}

The blade geometry is discretised into an unstructured triangular mesh generated using the Delaunay mesh generation tools in the partial differential toolbox (pdetool) of the commercial software MATLAB (www.mathworks.com). The final mesh, shown in figure 3, has 1380 elements and 928 nodes.

\subsection{FGM model of the blade geometry}

In order to construct the FGM model for the blade, the volume fraction of the material at the centroids of the triangular elements will have to be determined. Obviously the FGM variation should be such that the external surfaces of the blade are ceramic-rich to provide more resistance to heat transfer from the hot gases, while the material near the cooling passages should be metal-rich in order to aid the conduction of heat away into the cooling air. As shown in figure 2, the FGM region is taken to be the region between the external and internal surfaces of the airfoil while the region between the cooling passages themselves is assumed to be fully metallic.

An approximate procedure based on analytical geometry was used to calculate volume fractions of the material at the centroids of the triangular elements in the FGM region. The volume fraction of ceramic, $V_{c}$, varies as per the power law in (8). Distances $Z$ and $h$ are

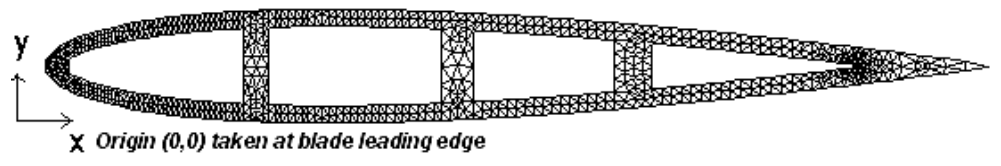

Figure 3. NACA0012 blade cross-section unstructured triangular mesh. 


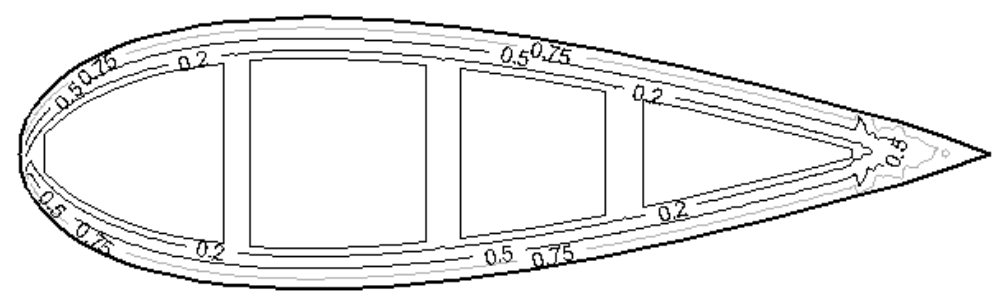

Figure 4. FGM model of airfoil with $V_{c}$ isolines of $0 \cdot 2,0 \cdot 5$ and 0.75 .

specially defined and computed from the element centroids to the fully-metal and fullyceramic surfaces that serve as reference surfaces for this FGM model (Coomar 2007).

$$
V_{c}=\left(\frac{Z}{h}\right)^{n}
$$

In (8) $n$ is the gradient index that controls the average volume fraction of the constituents. Figure 4 shows a zoomed-in view of the airfoil domain with isolines of ceramic volume fraction plotted for $V_{c}$ values of $0.20,0.50$ and 0.75 for a value of $n$ equal to $1 \cdot 0$. It can be seen that the FGM model mostly exhibits a smooth variation in material composition except for a few points near the trailing edge. Hence this FGM model is an accurate enough approximation which can be used for further analysis.

\subsection{Effective material properties}

Using the methods described in the previous section it is possible to obtain the volume fraction distribution over the domain of the blade for different values of $n$. Table 1 lists the average fraction of the metal and the ceramic in the domain for twelve selected values of the gradient index. The homogeneous fully ceramic blade corresponds to $n$ equal to zero, in which case even the regions between the cooling passages (see figure 2) are assumed to be totally ceramic.

Table 1. Average volume fraction of ceramic and metal.

\begin{tabular}{lll}
\hline $\begin{array}{l}\text { Gradient } \\
\text { index } n\end{array}$ & \multicolumn{1}{c}{$V_{c}$} & \multicolumn{1}{c}{$V_{m}$} \\
\hline 0 & 1 & 0 \\
$0 \cdot 1$ & $0 \cdot 7651$ & $0 \cdot 2349$ \\
$0 \cdot 2$ & $0 \cdot 7076$ & $0 \cdot 2924$ \\
$0 \cdot 3$ & 0.6569 & 0.3431 \\
$0 \cdot 5$ & 0.5718 & $0 \cdot 4282$ \\
$0 \cdot 7$ & 0.5036 & $0 \cdot 4964$ \\
$0 \cdot 8$ & 0.4744 & 0.5256 \\
1 & 0.4239 & 0.5761 \\
1.5 & 0.3306 & 0.6694 \\
3 & 0.1871 & 0.8129 \\
4 & 0.1397 & 0.8603 \\
20 & 0.0114 & 0.9886 \\
\hline
\end{tabular}


Table 2. Coefficients for metal and ceramic for use in (9) (Reddy \& Chin 1998).

\begin{tabular}{ccccc}
\hline Material & $k_{o}$ & $k_{1}$ & $k_{2}$ & $k_{3}$ \\
\hline Zirconia & 1.7 & $1.276 \mathrm{e}-4$ & $6.648 \mathrm{e}-8$ & 0 \\
Ti6Al4V & 1.0 & $1.704 \mathrm{e}-2$ & 0 & 0 \\
\hline
\end{tabular}

For a $n$ value of 20 , the blade is almost a fully metallic blade, since its ceramic content is less than $1 \%$.

In the FEM model, the temperature dependency of the thermal conductivity $k$ of both the metal and the ceramic is expressed in the form of:

$$
k=k_{o}\left(1+k_{1} T+k_{2} T^{2}+k_{3} T^{3}\right),
$$

where $k_{o}, k_{1}, k_{2}$, and $k_{3}$ are constants, and $T$ is in Kelvin. The ceramic chosen is Zirconia. Ti6A14V is the metallic component of the FGM. The values of the coefficients in (9) are listed in table 2. The simple rule of mixtures can then be implemented for effective material properties. Thus effective thermal conductivity of the FGM at any point in the domain is:

$$
k_{\mathrm{eff}}(T, x, y)=k_{m}(T)\left(1-V_{c}(x, y)\right)+k_{c}(T) V_{c}(x, y) .
$$

\subsection{Code development}

A FEM code was developed in MATLAB for simulating this heat transfer problem. For code validation in the absence of data representative of actual engine conditions, the FEM solution was compared to the exact solution for a hollow isotropic cylinder $0.3 \mathrm{~m}$ thick with outer radius $1 \mathrm{~m}$ and thermal conductivity of $30 \mathrm{~W} / \mathrm{mK}$ and the inner and outer surfaces experiencing convection at $425^{\circ} \mathrm{C}$ and $1000^{\circ} \mathrm{C}$ respectively, with $h$ equal to $500 \mathrm{~W} / \mathrm{m}^{2} \mathrm{~K}$ (Holman 2004). The simulated results were seen to match excellently with the analytical predictions as can be seen from table 3 .

Further, for the turbine blade simulation the agreement of the thermal energy balance, computed through the flux vectors and temperature gradients by from the FEM solution, was found to be within $\pm 2 \%$.

Table 3. Comparison of analytical and numerical results for hollow cylinder centered at $(0,0)$ subject to convection boundary conditions.

\begin{tabular}{lcc}
\hline Coordinates & Exact solution ${ }^{\circ} \mathrm{C}$ & FE solution ${ }^{\circ} \mathrm{C}$ \\
\hline$(0 \cdot 7,0 \cdot 0)$ & $523 \cdot 03$ & 522.95 \\
$(0 \cdot 8,0 \cdot 0)$ & 677.41 & $677 \cdot 36$ \\
$(0 \cdot 9,0 \cdot 0)$ & 810.98 & 810.94 \\
$(1 \cdot 0,0 \cdot 0)$ & $932 \cdot 01$ & 931.92 \\
\hline
\end{tabular}


Table 4. FEM solutions for FGM blade with TET of $1400 \mathrm{~K}$ and $T_{c}$ of $700 \mathrm{~K}$ and gradient index $0 \cdot 2$ on progressively finer meshes.

\begin{tabular}{lc}
\hline Number of elements & Mean blade temperature from FE solution ${ }^{\circ} \mathrm{C}$ \\
\hline 376 & 1073.23 \\
1080 & $1057 \cdot 65$ \\
1380 & $1040 \cdot 17$ \\
1878 & 1038.54 \\
2346 & 1037.46 \\
\hline
\end{tabular}

\section{Results and discussion}

A series of simulation runs for the FEM model for typical gas turbine operating conditions indicated that variation in the value of heat transfer coefficient had a much smaller effect on the blade temperature distribution when compared to the effect of changes in TET, coolant temperature $\left(T_{c}\right)$ and surface emissivity. For all the results presented in this study the coolant side heat transfer coefficient was therefore taken as $1200 \mathrm{~W} / \mathrm{m}^{2} \mathrm{~K}$ and the external heat transfer coefficient is governed by (2).

In order to confirm the suitability of the FGM model for the turbine blade, FEM simulation results on progressively finer meshes were obtained. These results are summarized in table 4 . It shows that the mean temperatures predicted for a TET of $1400 \mathrm{~K}$ and $T_{c}$ of $700 \mathrm{~K}$ in a functionally graded blade with index 0.2 are roughly the same as the mesh becomes denser. When the mesh has 375 elements, the model is not very accurate since the elements are too large and thus the volume fraction of the centroid are not representative of correct material volume fraction over the entire region of the element. The final mesh has 1380 elements and 928 nodes.

The FEM simulations in this study were carried out for two types of blades-(i) blades with FGM and (ii) blades with a layer of TBC. For each of the two types of blades, heat transfer with and without radiation was considered separately. The impact of variability in the TET, in the $T_{c}$ and in the external surface emissivity on the blade temperature distribution was quantified. For that purpose an index called cooling effectiveness is used. Cooling effectiveness is defined as the ratio of the difference in gas temperature (or TET) and the mean blade temperature (MBT) to the difference in gas temperature and cooling air temperature (or $T_{c}$ ) (Asok Kumar \& Kale 2002). The MBT is taken to be the mean of the temperatures evaluated at the centroids of all the triangles of the mesh.

\subsection{Temperature distribution of blades without radiation}

The results obtained from the FEM simulation with convection as the only external mechanism and no radiation are now discussed in detail. For the comparison of performance of FGM blades, six different values of the gradient index were chosen, namely, $0,0 \cdot 2,0 \cdot 7,1 \cdot 0,3 \cdot 0$ and 20 .

3.1a Effect of turbine entry temperature and material composition: The effect of increasing TET on the MBT for FGM blades exposed to only convective heat transfer with different gradient indices is shown in figure 5 .

It can be seen that MBT increases linearly with TET regardless of material composition. The rate of rise of MBT with TET is, on an average, about $0.48 \mathrm{~K} / \mathrm{K}$ for graded blades, while 


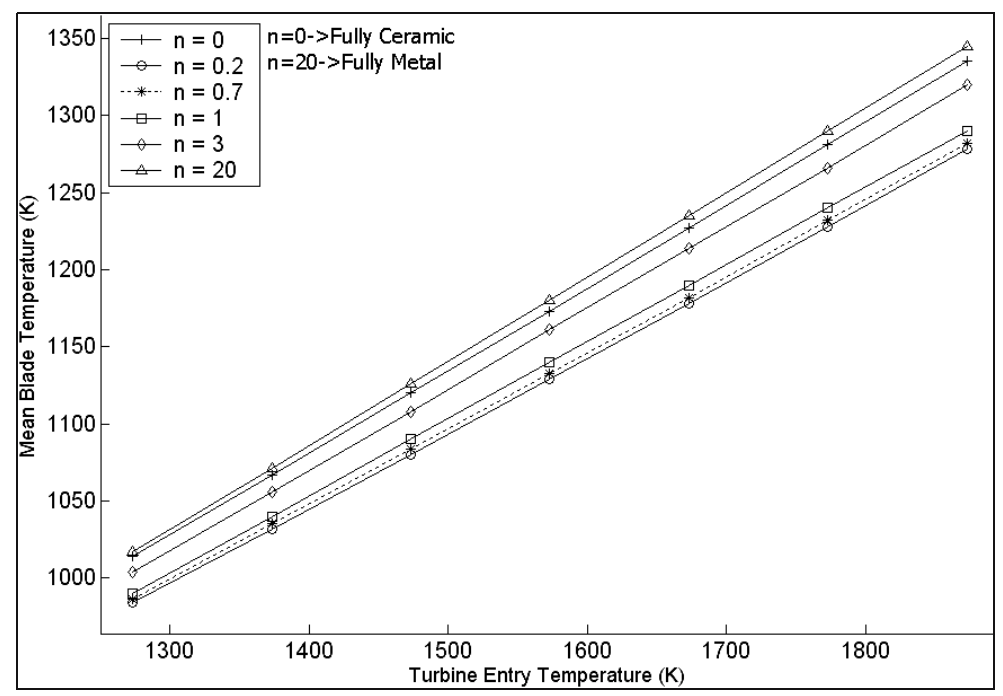

Figure 5. Effect of TET on mean blade temperatures (no radiation).

for almost homogeneous blades it rises at a slightly higher rate of about $0.53 \mathrm{~K} / \mathrm{K}$. As the gradient index increases, MBTs increase and temperature suppressions (difference between TET and MBT) consequently decrease. A similar trend is observed in figure 6 which plots cooling effectiveness against TET for different blades. Both figures 5 and 6 indicate that the functionally graded blade with index 0.2 is superior in performance to the other blades and experiences the lowest average temperature distribution. Also note that the homogeneous blades $(n=0,20)$ behave very similarly and do not show much difference in MBT.

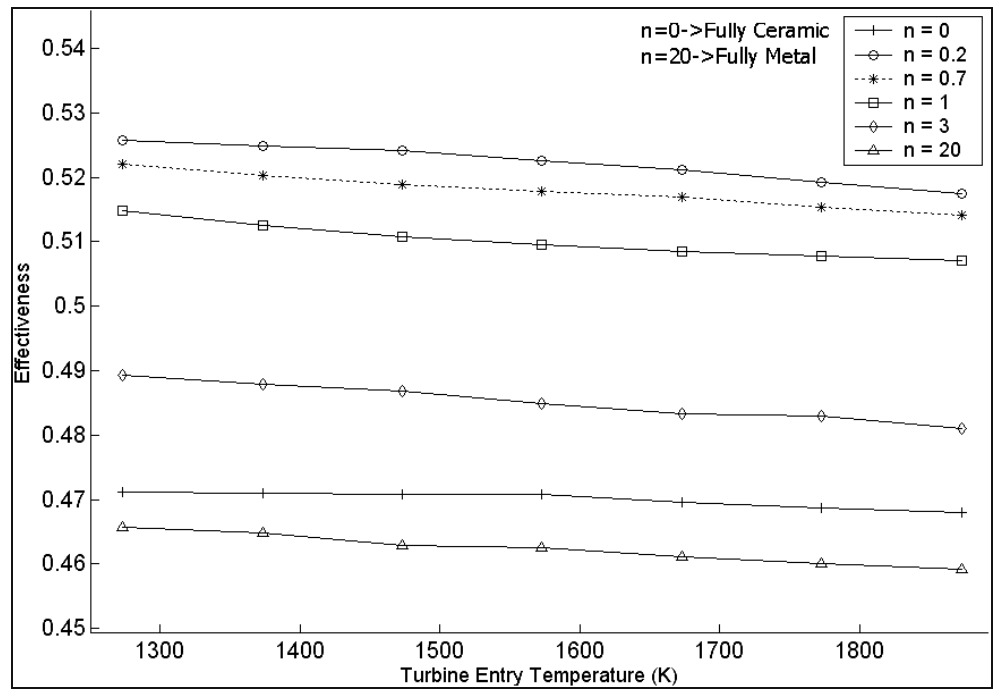

Figure 6. Effect of TET on cooling effectiveness (no radiation). 


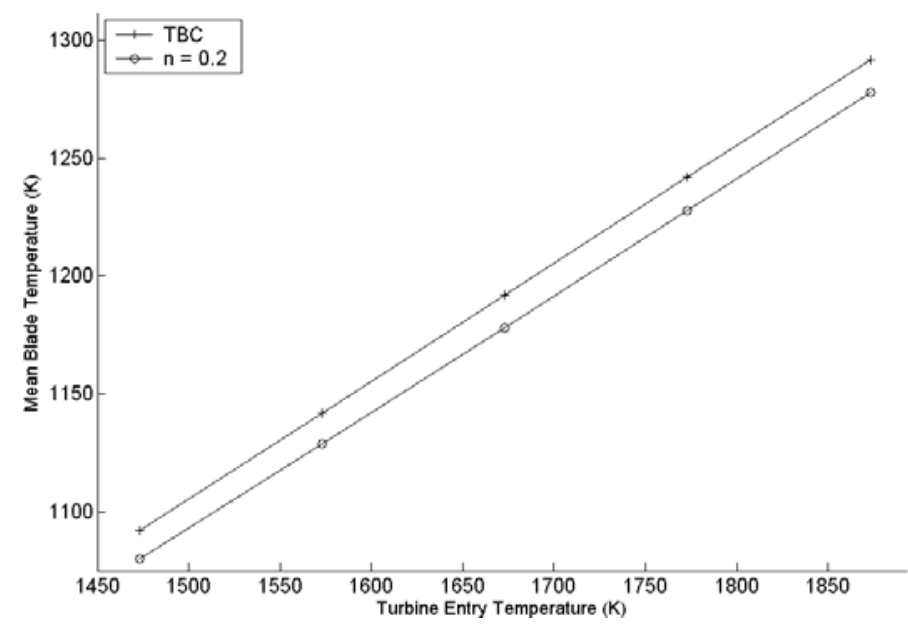

Figure 7. TET and MBT for FGM and TBC coated blade (no radiation).

We can now compare the FGM blade with index of $0 \cdot 2$ to a conventional metal blade with TBC coating also exposed to same convection conditions. The results of that comparison study are shown in figures 7 and 8 . From these plots it can be seen that on an average, the FGM blade runs as much as $12 \mathrm{~K}$ lower than its TBC counterpart. Thus the FGM blade compares favourably with the TBC blade as far as cooling performance is concerned and, in fact, betters it. This extra $12 \mathrm{~K}$ temperature suppression for the same TET that is now achievable using the FGM blade will allow design TETs to be pushed $12 \mathrm{~K}$ higher. This can lead to a $3 \%$ increase in turbine output and a possible increase of $1 \%$ in cycle efficiency (Boyce 2002).

3.1b Effect of coolant temperature and varying material composition: It will be pertinent to also examine the effects of an increase in the coolant air temperature (usually the temperature

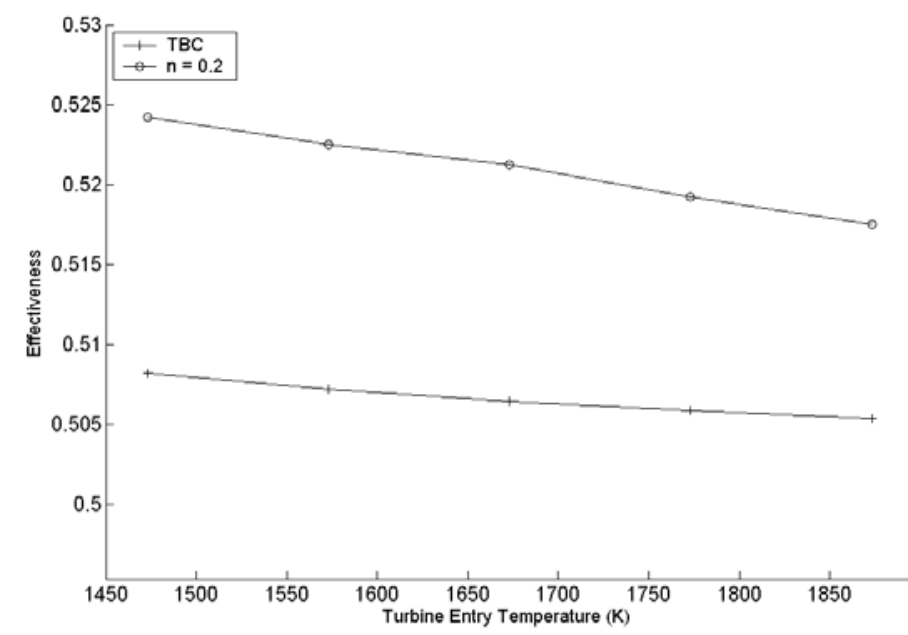

Figure 8. Cooling effectiveness for FGM and TBC coated blade (no radiation). 


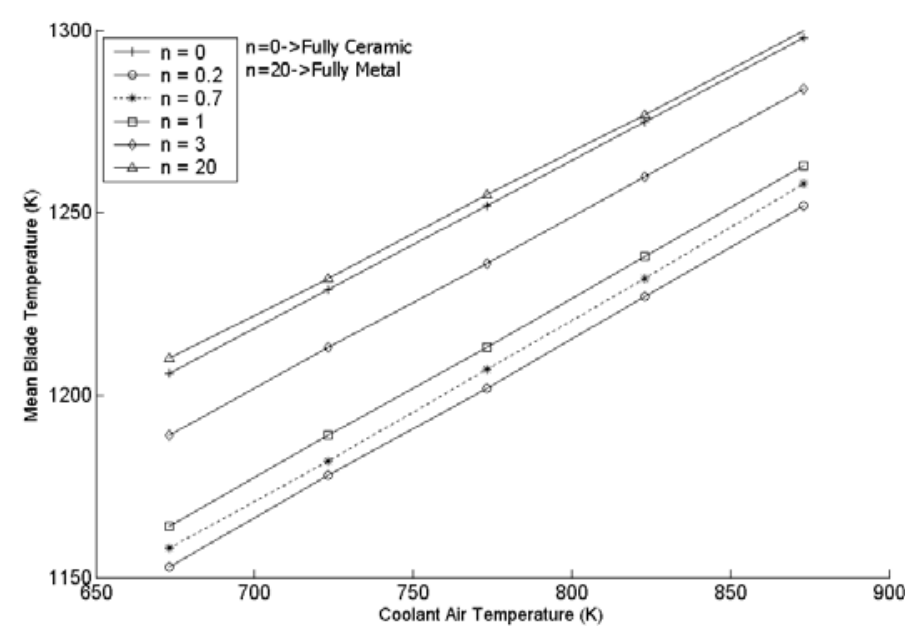

Figure 9. Coolant temperature and MBT for various blades (no radiation).

of the compressor discharge) on the overall temperature distribution in the blades. Simulations were therefore carried out for a range of $T_{c}$ values, with TETs fixed at the base value of $1673 \mathrm{~K}$. The results obtained are presented in figures 9 and 10. For all blades, MBTs increase linearly as $T_{c}$ goes up. As seen in figures 9 and 10, once again it is the functionally graded blade with index 0.2 that performs best under the adverse effect of an increase in $T_{c}$. The FGM blade with index 0.2 experiences temperatures that are $\sim 50 \mathrm{~K}$ lower on an average than temperatures experienced by materially homogeneous blades.

Looking at figures 5 and 9 we can also examine which of the two variables, the TET or the $T_{c}$, has a stronger effect on the mean blade temperatures. For different blade material compositions, there is an average rise of about $54 \mathrm{~K}$ in the MBT for a rise of every $100 \mathrm{~K}$ in TET. On the other hand, in response to a similar increase of every $100 \mathrm{~K}$ in $T_{c}$, MBTs on

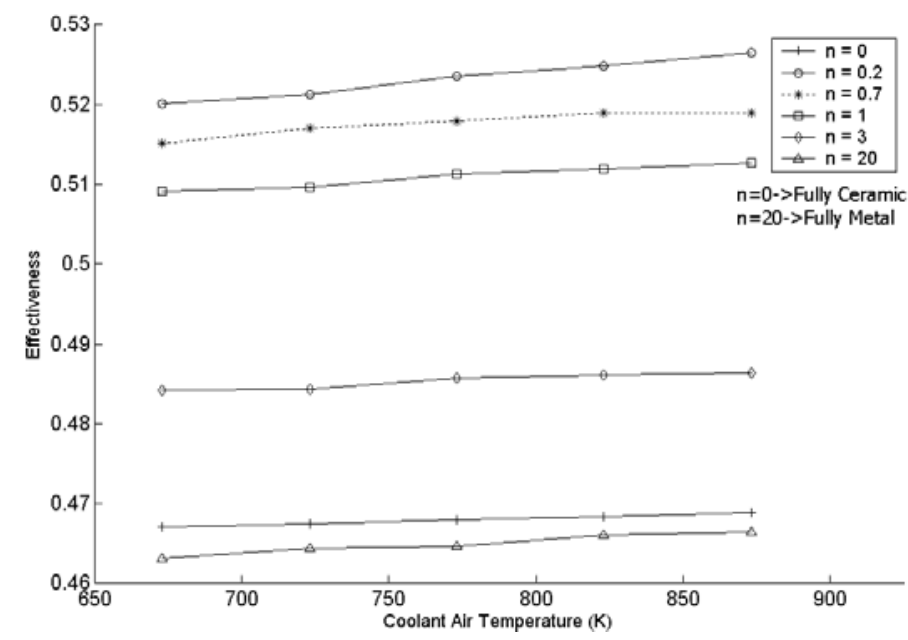

Figure 10. Effect of coolant temperature on effectiveness (no radiation). 
an average rise by about $44 \mathrm{~K}$. This demonstrates that, as far as MBTs of air colled turbine blades are concerned, it is the TET that has a bigger influence than the coolant temperature $T_{c}$. Interestingly, this is the reason why cooling effectiveness sometimes rises somewhat counterintuitively with increasing coolant temperature. The MBT responds much more slowly to increases in $T_{c}$ than to increases in TET, and so differences in TET and $T_{c}$ (the denominator) decrease much faster than differences in TETs and MBTs (the numerator), which can result in a net rise in numerical value of effectiveness. This manifests itself in figures 6 and 10 where the slopes of the lines in figure 10 can be seen to be less than the slopes in figure 6 . It is necessary to keep these points in mind so as not to construe that higher coolant temperatures are better for blade cooling.

\subsection{Temperature distribution of blades with convection and radiation}

Along with convection, the effect of radiation is now also included in the heat transfer model and the results are analysed.

3.2a Effect of inclusion of radiation heat load: Simulation results plotted in figure 11 help determine the degree to which radiation affects the temperature distribution of blades of different material compositions. A fully metal, a fully ceramic, a TBC-coated metal blade and a typical FGM blade $(n=0.7)$ are chosen for determining the effect of including radiation and the emissivity is taken as unity. Figure 11 clearly shows that on incorporating radiation the MBTs increase for all the blades by an average of about $70 \mathrm{~K}$. Clearly, radiation has a major impact on MBT regardless of material composition, and must therefore be part of any accurate heat transfer analysis, along with convection.

3.2b Effect of turbine entry temperature and material composition with radiation Functionally graded blades with various amounts of ceramic and metal content were then examined for changes in MBT due to changes in TET, this time with radiation included. Figure 12 summarizes the results for various blade compositions with emissivity value of 1 . A couple of conclusions can be readily made from the results shown in figure 12 . Firstly, once again, the FGM blade with index $0 \cdot 2$ experiences the lowest temperatures even when radiation is incorporated.

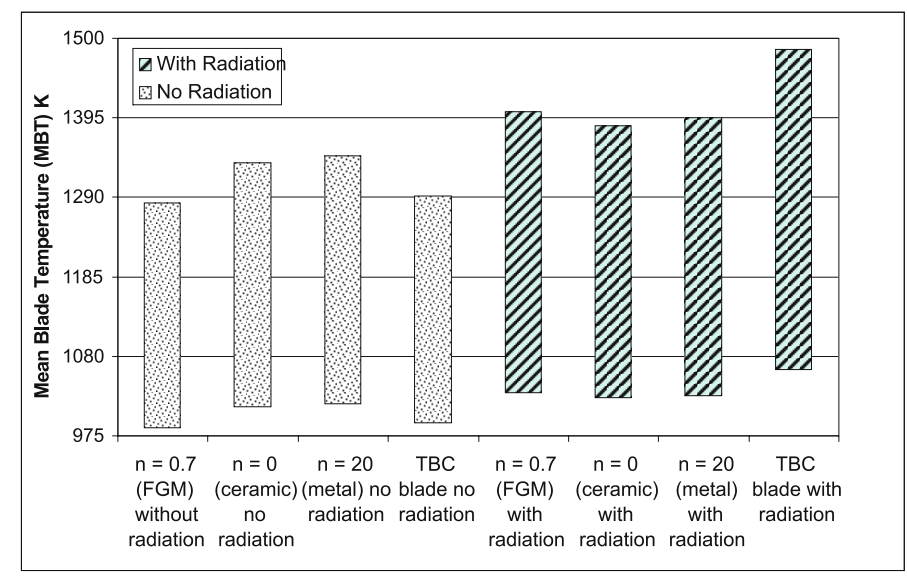

Figure 11. MBT Range experienced by blades with and w/o radiation and with external gas temperatures varying from $1273 \mathrm{~K}$ to $1873 \mathrm{~K}$. 


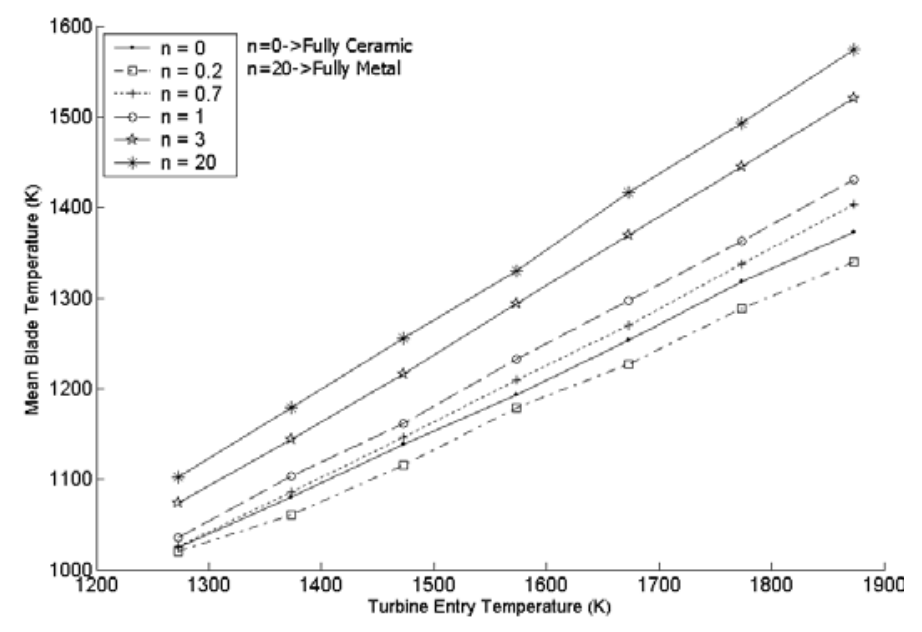

Figure 12. MBT variation with TET for FGM blades with black body radiation.

It delivers an average temperature suppression of $398 \mathrm{~K}$ which is about $20 \%$ better than the other FGM blades with index $n=0 \cdot 7,1$, and 3 . When compared to homogeneous blades, the temperature suppression is $40 \%$ more than fully metal blades and $7 \%$ more than fully ceramic blades. Secondly, once radiation is incorporated into the model, the fully ceramic blade comes out performing noticeably well while the fully metal blade fares much worse. MBTs for the fully ceramic blade are only $2 \%$ higher than MBTs for the graded blade with index $0 \cdot 2$. On the other hand, MBTs for the fully metal blade are about $13 \%$ higher. This situation thus differs from the results in section $3 \cdot 1$ a that had been obtained when only convection was considered. As pointed out in section $3 \cdot 1 \mathrm{a}$, both types of homogeneous blades, whether fully metal or fully ceramic, performed very much the same manner in the absence of radiation. This difference of thermal behaviour in including radiation merits a little discussion. It has already been shown in section $3 \cdot 1 \mathrm{~b}$ that TET has a stronger effect on MBTs than $T_{c}$. Since radiation heat load is proportional to the fourth power of TET, this stronger influence of TET on the MBT is further accentuated when radiation is incorporated. Physically, this implies that, as far as temperature suppression is concerned, the ability of the low-conductivity ceramic to block the radiation from the hot gases assumes more significance than the ability of the highconductivity metal to conduct heat away into the coolant passages. The fully ceramic blade, even though quite poor in letting the influence of the coolant air spread through its domain, is very effective in blocking radiation. On the contrary, the fully metal blade, though able to conduct heat away into the cooling passages effectively, suffers from a far greater handicap of not being able to block radiation. This is the reason that the fully ceramic blade performs so much better than the fully metal blade once radiation is incorporated.

\subsection{Effect of surface emissivity}

During operation, the gas turbine blade surfaces undergo changes due to deposition, corrosion and erosion. As turbine blades get older these deposits tend to blacken the surface so that its emissivity increases. Figure 13 shows how the MBTs of the blades increase as the surface emissivity takes on values from $0 \cdot 2$ to 1 while the TET is kept unchanged. The fully metallic blade is clearly the most sensitive to increases in emissivity. Also note that the FGM blade 


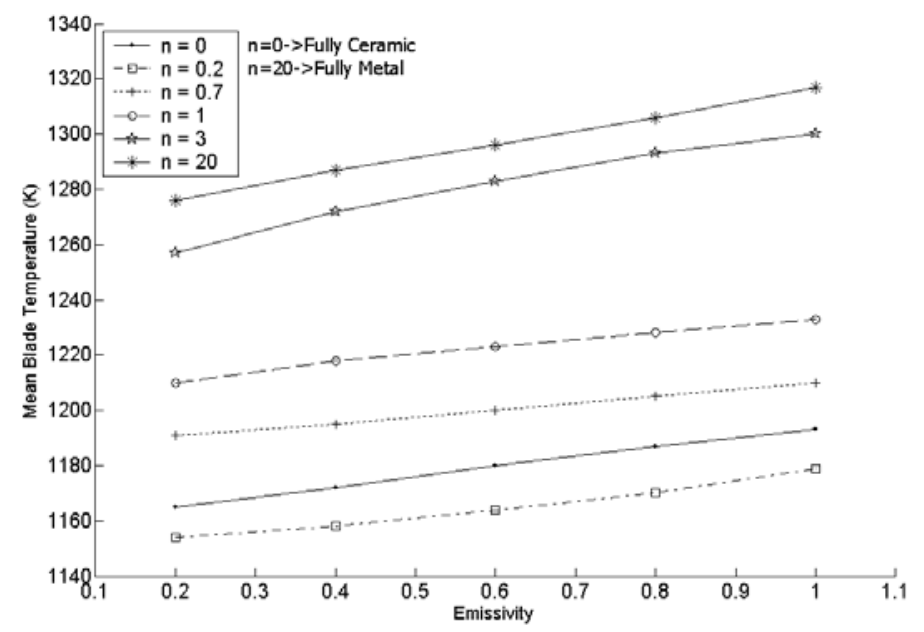

Figure 13. Effect of emissivity on MBT for various blades (TET = $1573 \mathrm{~K}$ ).

with index $0 \cdot 2$ experiences a rise of $25 \mathrm{~K}$ while the fully ceramic blade allows a similar $28 \mathrm{~K}$ rise in MBT. Both these blades are very effective in blocking radiation, unlike the fully metal blade.

It now remains to compare once again the performance of the FGM blade with index $0 \cdot 2$ with that of the metal blade with TBC and radiation included. As shown in figure 14 the FGM blade achieves MBTs that are, on an average, about $90 \mathrm{~K}$ lower than blades with TBC. Therefore, even after including the effect of radiation, the FGM blade achieves $22 \%$ more temperature suppression than the TBC blade over the entire range of TETs and emissivity values. This clearly indicates that the graded blade with index 0.2 can match, and indeed better the thermal performance of a conventional metal blade with TBC.

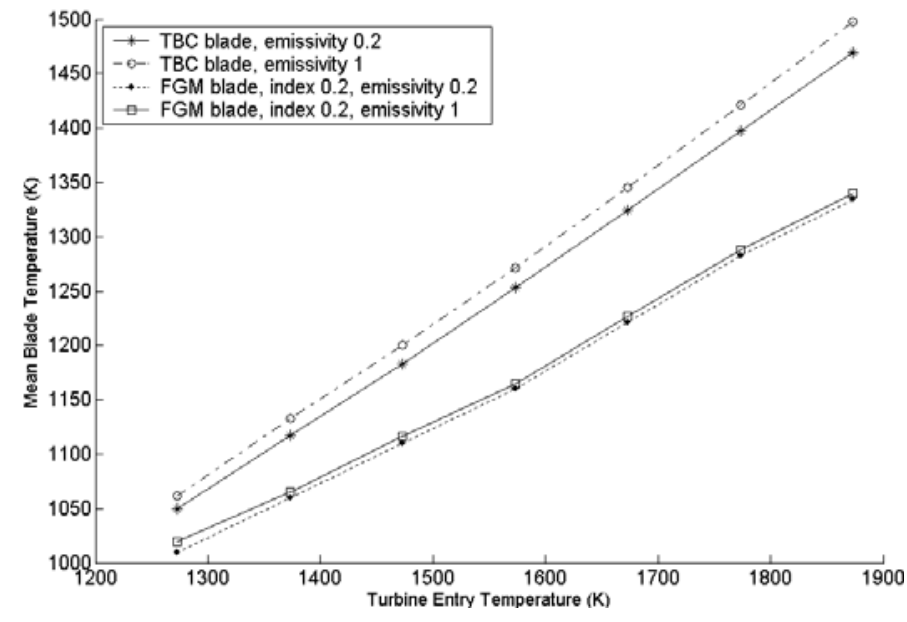

Figure 14. MBTs of TBC coated and FGM blade $(n=0 \cdot 2)$, with radiation. 


\section{Conclusion}

A procedure based on analytical geometry was implemented to draw up a model of a functionally graded turbine blade. Using the model, FEM simulations were carried out for obtaining temperature distributions for a range of turbine entry temperatures and various FGM compositions. Radiation was shown to be a significant factor in the heat transfer process. Effects of increasing TET, coolant temperature and surface emissivity were examined to determine which material composition yielded the best results under all the different types of thermal loading. Among all the FGM blades and the TBC blade, the graded blade with index 0.2 was found to perform better under a wide variety of input conditions irrespective of whether radiation was included or not. Since it is known that FGMs have smoother variation in material properties and are therefore less prone to debonding under thermal stresses. The results indicate that functionally graded air-cooled turbine blades can be a better alternative to TBCcoated metal blades for use in gas turbines.

\section{Nomenclature}

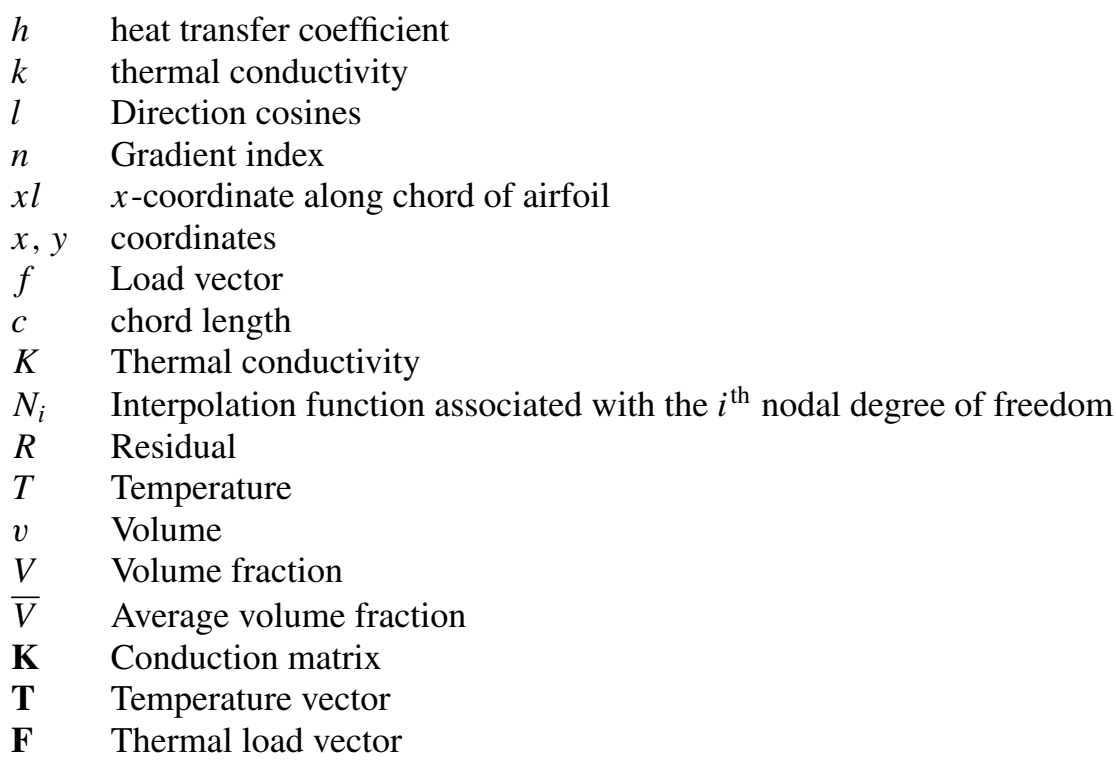

\section{Greek symbols}

$\begin{array}{ll}\varepsilon & \text { Emissivity } \\ \sigma & \text { Stefan Boltzmann constant } \\ \Omega & \text { domain }\end{array}$

\section{Subscripts}

$s, \Gamma \quad$ surface

$c \quad$ coolant gas

$\infty \quad$ surrounding fluid

$i \quad$ node designation

$r \quad$ radiation 


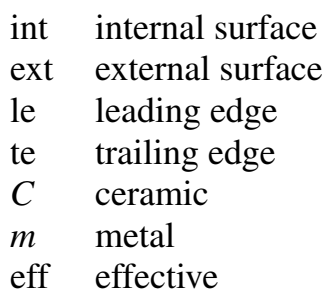

\section{Superscripts}

$e \quad$ element

\section{References}

Asok Kumar N, Kale S R 2002 Numerical simulation of steady state heat transfer in a ceramic-coated gas turbine blade. Intl. J. Heat and Mass Transfer. 45: 4831-4845

Aydin O, Iwata M, Arai N, Yang W J 2002 Theoretical analysis of heat transfer through an idealized gas turbine blade model with thermal barrier coating. Intl. J. Rotating Machinery. 8(2): 81-86

Berger J R, Martin P A, Mantic V, Gray L J 2005 Fundamental solutions for steady-state heat transfer in an exponentially graded anisotropic material. Zeitschrift fur angewandte Mathematik und Physik ZAMPZ. 56: 293-303

Boyce M P 2002 Gas Turbine Engineering Handbook. $2^{\text {nd }}$ Edition, (Boston: Butterworth Heinemann)

Bunker R S, Bailey J C, Ameri A A 2000 Heat transfer and flow on the first stage blade tip of a power generation gas turbine part 1: Experimental results. Trans. ASME J. Turbomachinery 122: 263-271

Carcasci C, Facchini B 1996 A numerical procedure to design internal cooling of gas turbine stator blades. Rev. Gén. Therm. 35: 257-268

Coomar N 2007 Numerical investigations of heat transfer in air cooled gas turbine blades, MTech Dissertation in Heat Power Engineering, National Institute of Technology Karnataka, Surathkal, India

Fujimoto T, Noda N 2000 Crack propagation in a functionally graded plate under thermal shock. Archive of Applied Mechanics. 70: 377-386

Garg V K 2002 Heat transfer research on gas turbine airfoils at NASA GRC. Intl. J. Heat and Fluid Flow. 23: 109-136

Holman J P 2004 Heat Transfer. $9^{\text {th }}$ Edition (New Delhi: Tata McGraw-Hill)

Huebner K H, Thornton E A, Byrom T G 1995 The finite element method for engineers. $3^{\text {rd }}$ Edition (Toronto: Wiley-Interscience)

Iacovides H, Launder B E 1995 Computational fluid dynamics applied to internal blade cooling: A review. Intl. J. Heat and Fluid Flow. 16: 454-470

Lakshminarayana B 1995 Fluid Dynamics and Heat Transfer of Turbomachinery. $1^{\text {st }}$ Edition, (New York: Wiley-Interscience)

Lee W Y, Stinton D P, Berndt C C, Erdogn F, Lee Y D, Mutasim Z 1996 Concept of functionally graded materials for advanced thermal barrier coating applications. J. American Ceramic Soc. 79(12): 3003-3012

Lewis R W, Nithiarasu P, Seetharmu K N 2004 Fundamentals of the Finite Element Method for Heat and Fluid Flow. (Sussex: John Wiley and Sons)

Oh S, Librescu L, Song O 2003 Vibration of turbomachinery rotating blades made up of functionally graded materials and operating in high temperature field. Acta Mechanica. 166: 69-87

Praveen G N, Reddy J N 1999 Thermoelastic analysis of functionally graded ceramic-metal cylinder. J. Eng. Mech. 125: 1259-1267

Reddy J N 1993 An introduction to the finite element method. $2^{\text {nd }}$ Editon (New York: McGraw-Hill) 
Reddy J N, Chin C D 1998 Thermomechanical analysis of functionally graded cylinders and plates. J. Thermal Stress 21: 593-626

Wang B, Mai Y, Zhang X 2004 Thermal shock resistance of functionally graded materials. Acta Materialia. 52: 4961-4972

Wang X, Bibeau E, Naterer G F 2007 Experimental correlation of forced convection heat transfer from a NACA airfoil. Exp. Therm. Fluid Sci. 31(8): 1073-1082

www.mathworks.com Partial Differential Equation Toolbox User's Guide, Version 1, The MathWorks Inc. 